\title{
MIDST: A System to Support Stigmergic Coordination in Data-Science Teams
}

\author{
Kevin Crowston \\ Jeffrey S. Saltz \\ Amira Rezgui \\ Yatish Hegde \\ crowston@syr.edu \\ jsaltz@syr.edu \\ arezgui@syr.edu \\ yhegde@syr.edu \\ Syracuse University \\ Syracuse, New York, USA
}

\author{
Sangseok You \\ you@hec.fr \\ HEC Paris \\ Jouy-en-Josas, France
}

\section{ABSTRACT}

We demonstrate MIDST, a system we developed to support stigmergic coordination in data-science teams, that is, coordination supported by a shared work product. To improve coordination, the system supports modularization of an analysis as a workflow, distributed code development and sharing and tracking of task status through a web application.

Permission to make digital or hard copies of all or part of this work for personal or classroom use is granted without fee provided that copies are not made or distributed for profit or commercial advantage and that copies bear this notice and the ful citation on the first page. Copyrights for components of this work owned by others than ACM must be honored. Abstracting with credit is permitted. To copy otherwise, or republish, to post on servers or to redistribute to lists, requires prior specific permission and/or a fee. Request permissions from permissions@acm.org.

CSCW' 19 Companion, November 9-13, 2019, Austin, TX, USA

() 2019 Association for Computing Machinery.

ACM ISBN 978-1-4503-6692-2/19/11_..\$15.00

https://doi.org/10.1145/3311957.3359509 


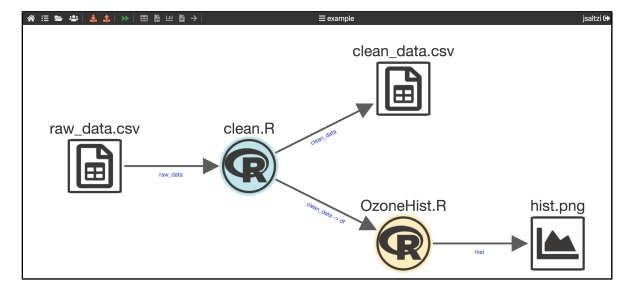

Figure 1: Network view showing data flowing between nodes

\section{CCS CONCEPTS}

- Human-centered computing $\rightarrow$ Computer supported cooperative work; Asynchronous editors; Empirical studies in collaborative and social computing; • Information systems $\rightarrow$ Data analytics.

\section{KEYWORDS}

stigmergic coordination, translucency, awareness, data-science teams

\section{ACM Reference Format:}

Kevin Crowston, Jeffrey S. Saltz, Amira Rezgui, Yatish Hegde, and Sangseok You. 2019. MIDST: A System to Support Stigmergic Coordination in Data-Science Teams. In 2019 Computer Supported Cooperative Work and Social Computing Companion Publication (CSCW'19 Companion), November 9-13, 2019, Austin, TX, USA. ACM, New York, NY, USA, 4 pages. https://doi.org/10.1145/3311957.3359509

\section{INTRODUCTION}

Data science is an emerging discipline that combines expertise across a range of domains, including software development, data management and statistics. Data-science projects typically have a goal of identifying correlations and causal relationships, classifying and predicting events, identifying patterns and anomalies and inferring probabilities, interest and sentiment [2]. A common data-science tool is R [1]: analyses are performed by writing programs in the R language, which typically take data as input and output analysis results. While small analyses can be performed by an individual, larger projects require a team of analysts working together. We will demonstrate a system to support coordination in data-science teams.

\section{SYSTEM OVERVIEW}

MIDST (Modular Interactive Data Science Tool) is a web-based data-science application that was developed for this project. The tool enables a team of data scientists to collaborate on developing an analysis, which is implemented in R. MIDST has three integrated views that team members use to create an analysis (or part of an analysis): the network, task and code views.

Network View. The main view of the analysis is as a workflow, in MIDST's network view. As with other data-flow tools, the network view helps users break an analysis into smaller chunks of work (nodes), and then visualize the flow of data through the nodes that comprise the analysis. There are three kinds of nodes: executable nodes that contain R code (code modules), data nodes that can be connected to an input of a node and visualization nodes that can be connected to an output of a node. For example, Figure 1 shows a simple analysis that reads in a raw data file (the raw_data.csv node), cleans and saves the data file (the clean. $R$ code node outputting to the clean_data.csv data 


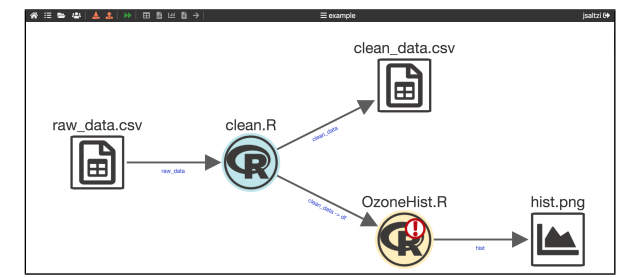

Figure 2: Network view after execution showing nodes with errors

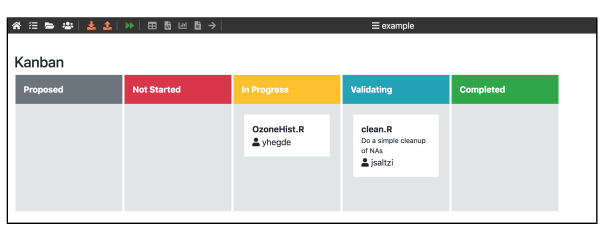

Figure 3: Task status view, showing status of work on each node

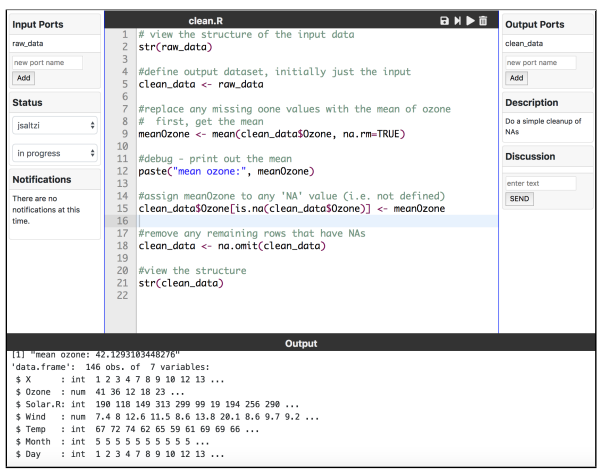

Figure 4: Edit interface for code in a node, showing code, input and output ports and execution output node) and generate a histogram (the OzoneHist. $\mathrm{R}$ code node reading from the clean. $\mathrm{R}$ node and outputting to the hist.png visualization node).

In the network view, users can add new nodes, define a node's inputs and outputs and connect nodes together, implementing a flow of data between the nodes. As users update the network (e.g., adding nodes or connections), the changes are propagated to other users viewing the network. Users can execute the entire network in the network view by pressing the 'Run' button at the top of the network view window. Any errors that occur during execution of the network are visible as failed nodes, shown by the exclamation mark in Figure 2. Other controls push or pull code changes or change views (discussed below).

Task View. A second view of the nodes is a task view, shown in Figure 2. Similar to other task boards, such as Trello (www.trello.com), the status of each code node is indicated by the column it appears in. Users can update the status of a node by simply dragging it to a new column. Tasks (i.e., nodes) can also be created in this view, which will add them to the workflow, but without connections. MIDST's task view provides a quick overview of the project status: what is being worked on, who is working on it and the overall balance between completed and uncompleted work. Status is also shown in the network view (Figure 1): each module is colored according to its status. MIDST provides hints to help a team member keep the status of their nodes up-to-date. For example, when someone starts to edit a node, the system suggests that it be moved to "in progress" status; when a user pushes code to share it with other team members, MIDST asks if that node's status should be moved to "validating".

Code View. Third, by clicking twice on a code node, within either the network or task view, a user drills down to the $\mathrm{R}$ code for the node. (Clicking on a data or visualization node gives a preview.) An example is shown in Figure 4, which happens to be the $\mathrm{R}$ code for the clean module from Figure 1. In this view, similar to how one uses RStudio, which is a popular interactive development environment for creating $\mathrm{R}$ applications, the user writes, edits, runs and debugs $\mathrm{R}$ code to implement the required functionality for the node. Within the code editor, a user can run the entire node or execute a single line of code (e.g., for debugging). Output from execution is shown in the bottom window pane of the code editor. This output is for the most recent run of the node, whether that was due to the full network being run in the network view, the full node being run in the code editor view, or a specific line being executed within the code editor.

The node's input ports and output ports (i.e., the connections to other nodes) are shown on either side of the code. An automatically-generated $\mathrm{R}$ preamble reads the input ports and makes the data available to the user's R script as variables with the same name; a postamble takes the contents of the named variables and adds them to the output ports to transfer to other nodes. The author of the $\mathrm{R}$ code is responsible for making the connection between these input and output variables. 


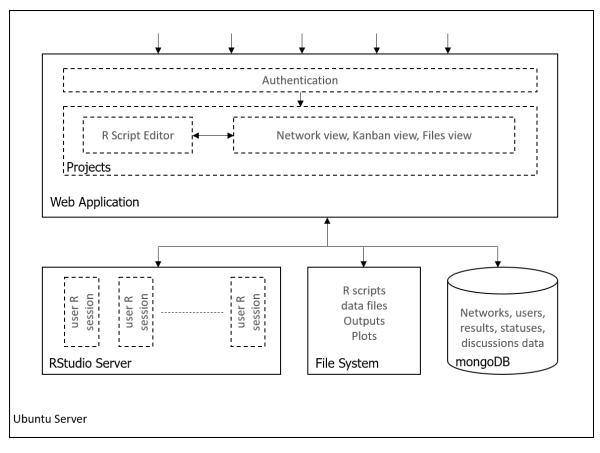

Figure 5: MIDST System Architecture
Figure 4 shows other collaboration features within the editor. For example, via the status widget on this view, a user can update the status and owner of a node. Team members can post messages about a node in the discussion widget. The MIDST system also posts messages here, e.g., to inform a user when the code being viewed is out-of-date (i.e., that another team member has shared a more recent version of the code)

Code sharing. The code in a code node is shared with other users viewing the project. In contrast to the network view that refreshes automatically for every user change, users must explicitly share changes to code, either for one module or the entire network, using a graphical code management system that lets them "push" their updates to and "pull" others' updates from a centralized code repository. The difference between these two approaches is because as a user edits code, it is likely that their code will often be in an intermediate, non-working condition. If such changes were pushed as they are made, other users would often find that they could no longer run the entire network, which could block their own work ("breaking the build"). Instead, users can wait until their code is in a usable state before sharing it and defer accepting others' changes until they are ready to incorporate them, since changes could potentially require them update their own code to fit.

Shared Execution Environment. To avoid incompatibilities between different users' computers, MIDST provides access to a common computing infrastructure, where each user has a clone of the same computing environment. More specifically, the current implementation is a web application, meaning that all the code runs on a common server. The system architecture is shown in Figure 5. The shared execution environment greatly facilitates team collaboration since issues such as what libraries and what versions are installed as well as other details such as location of data files are eliminated.

\section{ACKNOWLEDGMENTS}

This work was partially supported by a grant from the US National Science Foundation, IIS 16-18444.

\section{REFERENCES}

[1] [n.d.]. The R Project for Statistical Computing. https://www.r-project.org/

[2] M. Das, R. Cui, D. R. Campbell, G. Agrawal, and R. Ramnath. 2015. Towards methods for systematic research on big data. In 2015 IEEE International Conference on Big Data (Big Data). 2072-2081. https://doi.org/10.1109/BigData.2015.7363989 